
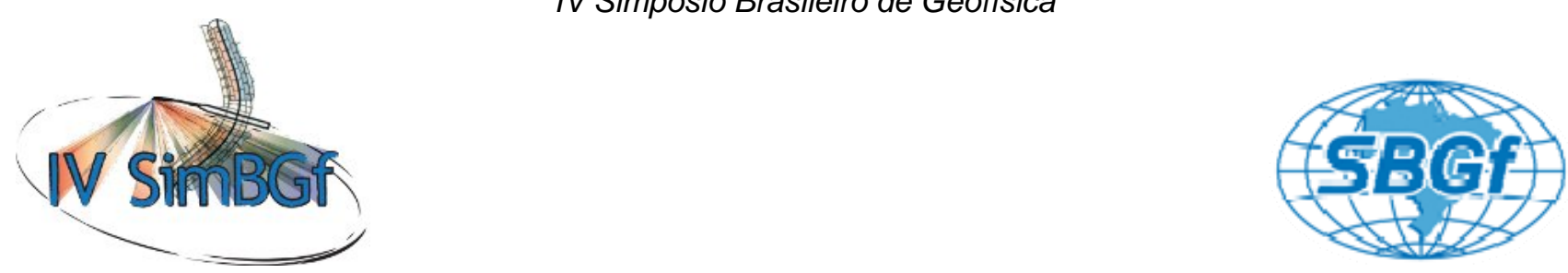

\title{
Análisis de la velocidad de reemplazamiento variable.
}

Gonzalo Pedraza, Estudiante Maestría Geofísica Universidad Nacional de Colombia. Prof. Dr. Castillo López Luis Antonio, Profesor Universidad Nacional de Colombia. Carlos Pedraza, Analista de Procesamiento Senior, Petroseis Ltda.

Copyright 2010, SBGf - Sociedade Brasileira de Geofísica

Este texto foi preparado para a apresentação no IV Simpósio Brasileiro de Geofísica, Brasília, 14 a 17 de novembro de 2010. Seu conteúdo foi revisado pelo Comitê Técnico do IV SimBGf, mas não necessariamente representa a opinião da SBGf ou de seus associados. É proibida a reprodução total ou parcial deste material para propósitos comerciais sem prévia autorização da SBGf.

\begin{abstract}
Onshore seismic surveys includes some considerations Statics into seismic processing sequence, for instance, the replacement velocity used for corrections of topography, elevations or weathering layer. This documents try the replacement velocity like an approach to a variable model, although in conventional processing this velocity comprise an unique value for all the area. Then it is possible obtain a model with variable velocity, including the weathering or low velocity layer effects.
\end{abstract}

\section{Introducción}

En geofísica se hace necesario recurrir a modelos que faciliten la imagen para definir la disposición del subsuelo, por lo que se busca encontrar una solución mediante la creación de sintéticos, iniciando con modelos y parámetros lo más próximos a la realidad. Más que tratar de replicar la realidad, el nivel de complejidad del modelo depende de los requerimientos en exactitud de los resultados y el avance técnico que se disponga en ese momento. Este documento es elaborado con el fin de abordar los modelos de correcciones estáticas en sísmica de reflexión, para generar un modelo con correcciones estáticas a partir de una velocidad de reemplazamiento variable. A la vez se recopila información acerca del datum utilizado para estas correcciones.

Cuando se trabaja información sísmica de reflexión en casos de topografía abrupta, las imágenes sísmicas presentan dificultades en el procesamiento de esta información, obteniendo imágenes con poca resolución, e imprecisas. Estos problemas surgen a partir de la aproximación que se debe realizar en el modelo de correcciones estáticas, ya que, en la mayoría de los algoritmos se necesita un valor único de datum y velocidad de reemplazamiento. En los casos en los que la topografía es abrupta estos modelos no son bien aproximados.
Existen diferentes estrategias para solucionar este inconveniente tales como tomografías de refracción, filtrado de información, continuación del campo de onda, etc. Si se tiene en cuenta que cuando hay cambios de topografía las condiciones de la capa meteorizada también cambian y que esta capa no es homogénea, es claro que los modelos de correcciones estáticas que utilizan la velocidad de reemplazamiento constante no tienen en cuenta estos fenómenos que están relacionados con las características geológicas del área.

En áreas con topografía abrupta, los tiempos de reflexión son afectados, ocasionando en algunos casos pérdidas de continuidad de los eventos y en otros la formación de estructuras que no son reales.

Una posibilidad de incluir los efectos de erosión y cambios de la capa meteorizada, es el considerar un modelo con velocidad de reemplazamiento variable.

\section{Correcciones Estáticas}

Las correcciones estáticas permiten determinar los tiempos de arribo de las reflexiones que podrían haber sido observados si todas las medidas se hubieran hecho sobre un plano horizontal sin presencia de capa meteorizada o del material de baja velocidad (Sheriff, 1991). Estos corrimientos en tiempo aplicados a las trazas sísmicas para compensar los efectos de variaciones en elevación, espesor y velocidad de la capa meteorizada, son llevados a un datum o nivel de referencia.

Los algoritmos de procesamiento tales como el análisis de velocidad, NMO, y apilado, son basados en la suposición que los datos fueron registrados sobre una superficie plana (Bevc, 1997).

Enfoques para las correcciones estáticas: existen dos formas de calcular esta corrección (Oria, 2002). Una de ellas es utilizando primeros arribos y la otra es mediante la adquisición de datos sísmicos de refracción. 


\section{Evolución de las capa de reemplazamiento}

Aunque se pueden encontrar diferentes trabajos relacionados con el tema han sido escogidos algunos trabajos que contienen elementos esenciales para el desarrollo de las correcciones estáticas y en especial de la capa de reemplazamiento.

La utilización de una capa con velocidad de reemplazamiento es fundamental en los resultados de la sísmica de reflexión (figura 1), aunque para la época este resultado debió ser fabuloso y lo sigue siendo, puede perder importancia ya que este tipo de problemas se solucionan con algunas herramientas de proceso.
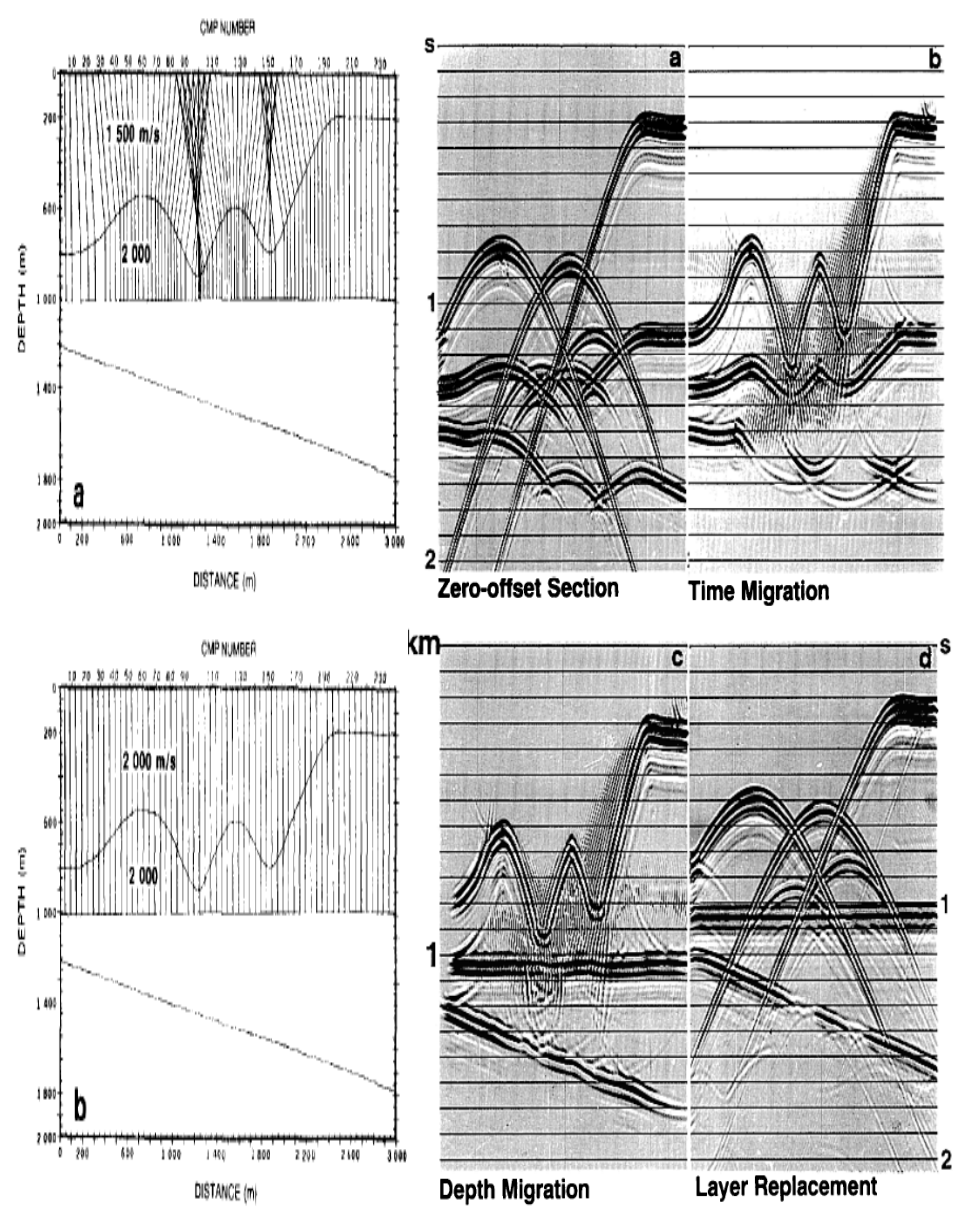

Figura 1. La diferencia en la eleccion del valor de la capa de remplazamiento es notable para este ejemplo, figura a y $b$ de la parte izquierda (Tomado de Yilmaz, 1986).

Sin duda en este caso se quiere mostrar la importancia de utilizar una capa con una velocidad de reemplazamiento para las correcciones por topografía. Las figuras ( $\mathrm{c}$ y d) en la parte derecha, muestran los resultados del reflector buzante con la capa de reemplazamiento idéntica al modelo geológico expuesto y por no decirlo con mayor definición que el resultado con la migración en profundidad.
A medida que pasan los años se ve que es adoptada esta idea, se trabaja una velocidad de reemplazamiento y se toma como estándar para la solución de problemas con topografía. Sin embargo, los problemas que generan en el momento de la elección de la magnitud de la velocidad de la capa de reemplazamiento se hacen notar y se continúa la investigación; ya es suficiente la utilización de una velocidad de reemplazamiento única, pues no siempre la solución es la esperada y se trabaja en este sentido, pues la propagación de los rayos no es la adecuada.

De esta forma se comienza a hablar de continuación del campo de onda para darle una mayor aproximación a la solución a los problemas ocasionados por la topografía y capa de baja velocidad. Este modelo es una modificación del anterior pero en el cual la propagación de los rayos no es de una forma abrupta, simulando que los rayos llegan a los detectores en ángulo recto (Figura 2), la capa posee un modelo de velocidad en profundidad la cual propaga los rayos de una forma no lineal.

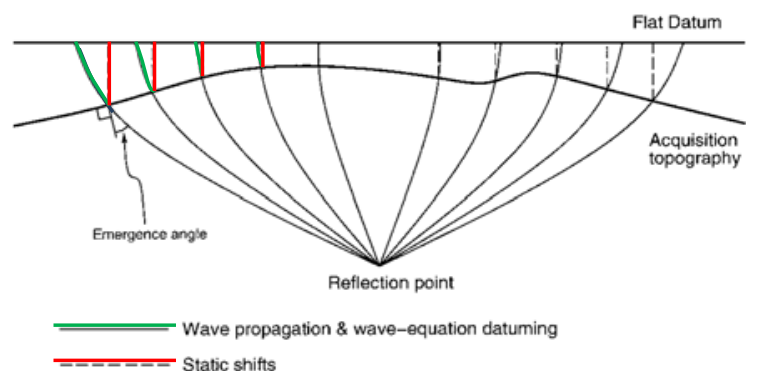

Figura 2. Los rayos son curvos con el nuevo modelo de correcciones estáticas (Tomado de Bevc, 1997).

Un ejemplo de una metodología en la que la interpretación comienza desde que se realiza la adquisición y no con los resultados migrados, es el que debe incluir la interacción maquina-analista. Aunque para este trabajo la interpretación de los resultados de la capa de baja velocidad es analizada con sísmica de refracción. Puede observarse como influencian los resultados en una sección sísmica. Este trabajo trata de extraer no sólo la utilización de una capa con velocidad de reemplazamiento sino la forma y sus variaciones laterales, se puede ver (figura 3) el mismo modelo de una capa meteorizada la cual ha sido erosionada por un río y ha dejado expuesta un nuevo tipo de roca, a su un resultado de la misma capa pero con métodos de interpolación, el cual se obtiene un resultado geológicamente errado. 

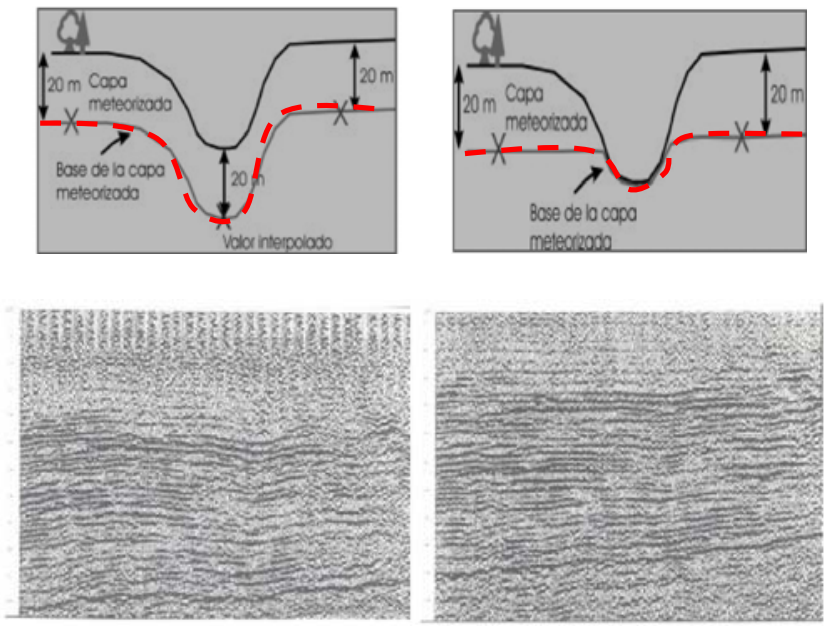

Figura 3. En esta figura se representa las diferencias ocasionadas por un mal modelo de la capa meteorizada y su respectiva velocidad de reemplazamiento (Tomado de Oria, 2002).

Los últimos trabajos relacionados con el tema están encaminados a combinar el enfoque de la capa con una velocidad de reemplazamiento e incluir los efectos que se ocasionan cuando la topografías es abrupta y se presentan diferentes capas aflorando en la superficie, pues de este modo, el modelo que se utiliza de la capa de reemplazamiento no se ajusta al modelo, en algunas ocasiones la magnitud de la velocidad de esta capa es un valor promedio entre el sustrato y la capa de baja velocidad, obteniendo un único valor, en otros casos simplemente se escoge un valor utilizado en algún otro proyecto de la misma cuenca.

En la literatura de geofísica se encuentran intentos por trabajar esta temática con únicamente datos de sísmica de reflexión, y utilizando herramientas de procesamiento de software especializados i.e., ProMax. En Castillo y Pedraza (2002), se cuestiona la efectividad de trabajar las correcciones estáticas en la secuencia de procesamiento con una velocidad y no un modelo con velocidad de reemplazamiento variable, sin embargo los resultados no han sido validados. Se ejemplifican con registros y las variaciones de velocidad en superficie y los resultados que se pueden obtener al utilizar en las correcciones estáticas una capa de reemplazamiento variable.

\section{Resultados preliminares}

Se tienen dos estrategias de trabajos para crear el modelo de reemplazamiento variable. Como base se trabaja con diferentes modelos sintéticos en los que se representa un modelo geológico que contiene una topografía abrupta y cambios de velocidades en superficie ocasionados por diferentes afloramientos.

Mediante trazado de rayos se realiza una simulación de una adquisición sísmica. Con ella se trabaja para ver el comportamiento del modelo de velocidad de reemplazamiento variable.

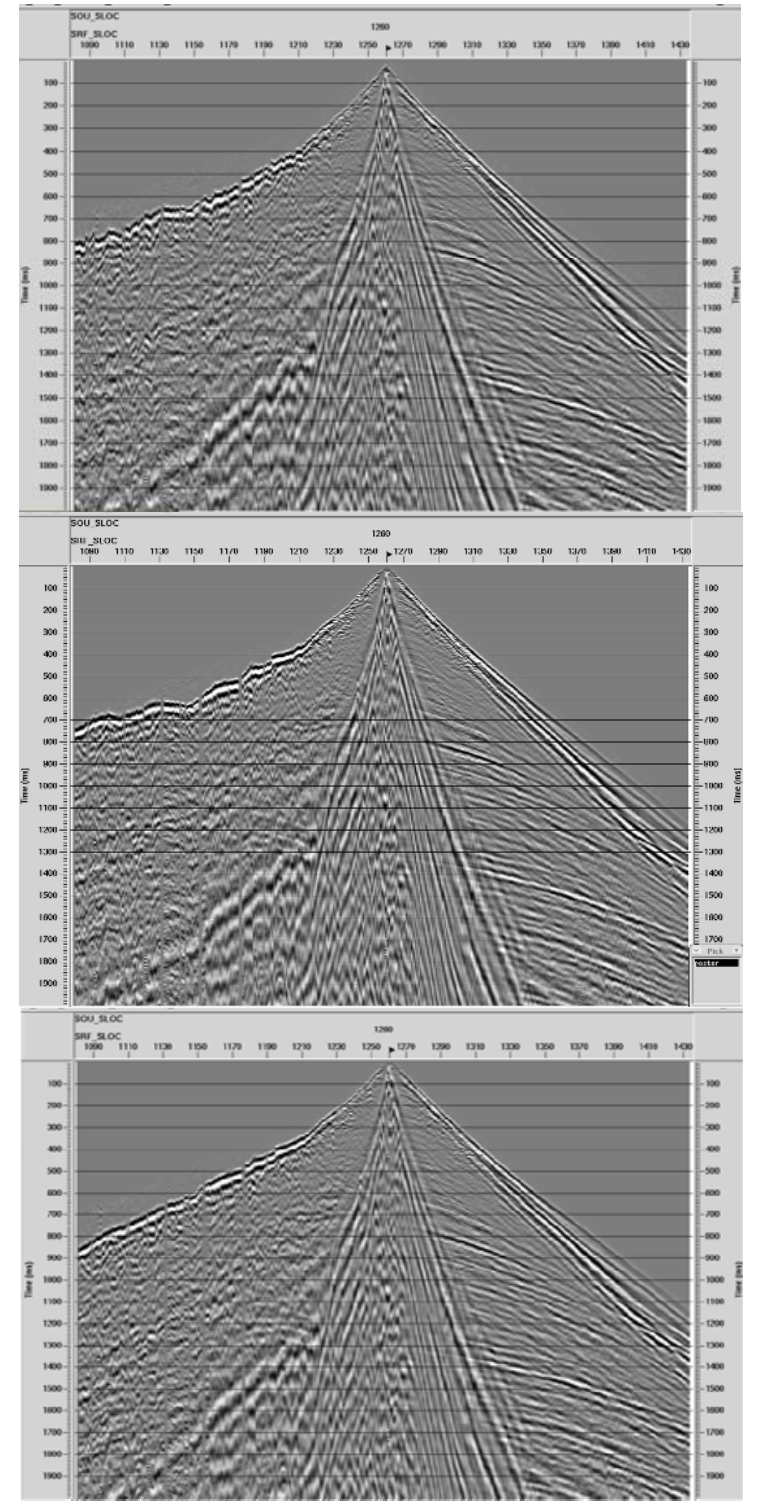

Figura 4. La imagen en la parte intermedia es corregida con un valor de 2100 y la de la parte inferior es corregida con una velocidad de reemplazamiento variable.la imagen de la parte superior es el registro sin correcciones (Tomado de Mora, 2009).

Por otro lado se toma como variable el datum y la influencia de este en la adquisición ya que el valor como se ha expuesto, es un parámetro que pasa desapercibido dentro de las correcciones estáticas, para ello se realiza otra simulación directamente en un valor de datum (línea roja, figura 5), la cual es comparada con otra que se realiza con un datum que es variable (línea verde, figura 5 derecha), en la que se puede ver claramente que esa nueva superficie favorece la convergencia de los rayos. 

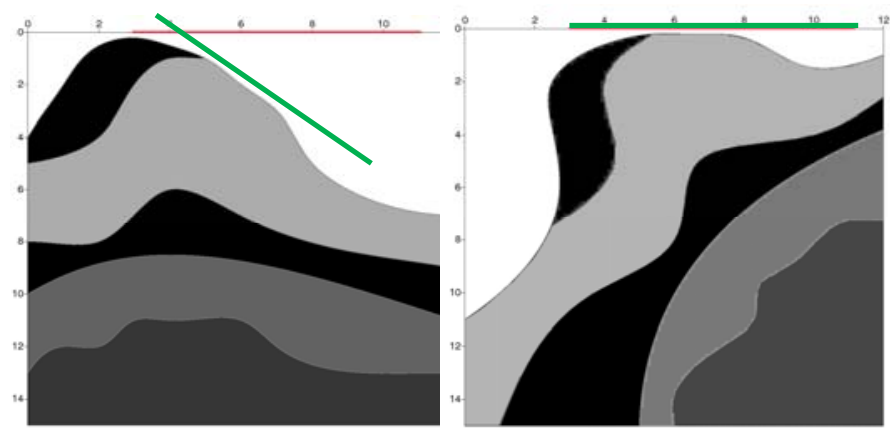

Oria, D, S. Dos Santos, 2002. Metodologia para la elaboracion de modelos de capa meteorizada. XI congreso Venezolano de geofisica, Caracas.

Sheriff, D. E., 1991. Encyclopedic Dictionary of Exploration Geophysics. Third Edition. Society Exploration Geophysics.

Yilmaz, O, \& Lucas, D., 1986. Prestack layer replacement, Geophysics Vol. 51, No. 7, pag. 13551369.

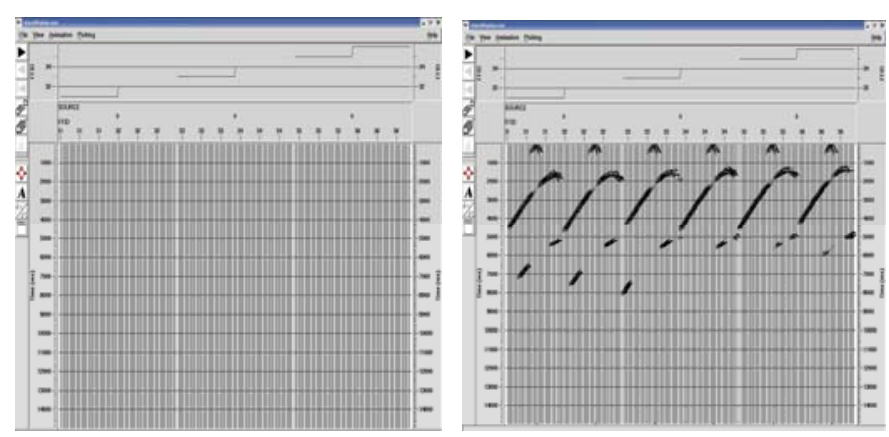

Figura 5. En esta figura se presentan el mismo modelo geológico en el que se realiza una simulación de una adquisición sísmica, en el de la derecha se le realiza una rotación para suponer que la adquisición se hace en un datum variable respecto al modelo de la izquierda.

En este ejemplo podemos mostrar que no solo la capa de reemplazamiento es necesaria si no que la elección de del datum es crucial, si se ve la sísmica de reflexión simplemente como un problema de óptica geometría el datum debería ser una valor asociado a una geométrica determinada por las capas y no simplemente un valor.

\section{BIBLIOGRAFÍA}

Bevc, D. 1997. Flooding the topography: Wave-equation datuming of land data with rugged acquisition topography, Geophysics, vol. 62, No. 5 (September-October); p. 1558-1569, 18 figs.

Castillo, L. \& Pedraza, C. 2002. Solución a un problema estático en un área con topografía abrupta usando una velocidad de reemplazamiento variable. Primer Simposio Colombiano de Sismología.

Mora, J. \& Pedraza, C. 2009. ¿Qué tan acertado es el uso de una función constante de velocidad de reemplazamiento en el procesamiento de datos sísmicos?. X Simposio bolivariano exploración petrolera en las cuencas subandinas. 